\title{
Targeted pain relief for dentine hypersensitivity
}

Colgate has launched an innovative addition to the Colgate Sensitive Pro-Relief range the Colgate Sensitive Pro-Relief Sensitivity Relief Pen. Containing Pro-Argin technology, it offers instant targeted pain relief right at the source of pain (achieved with direct

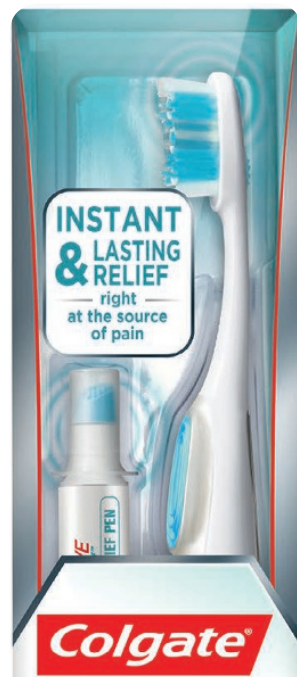

application of toothpaste massaged on the sensitive tooth for one minute). The pen comes with a soft bristled toothbrush ideal for sensitivity sufferers and is stored neatly within the toothbrush handle.

The Colgate Sensitive Pro-Relief Sensitivity Relief Pen is easy to use; simply twist the top of the pen to release a gel into the applicator tip and then directly apply to the affected tooth.

\section{SENSITIVE}

\section{PRO-Relief}

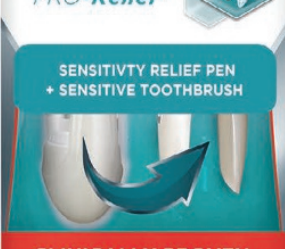

CLINICALLY PROVEN
The gel is clinically proven to provide instant relief from dentine hypersensitivity (for instant relief, apply the gel directly to the sensitive tooth and massage gently for one minute)

Pro-Argin technology, available also in Colgate Sensitive Pro-Relief toothpastes, contains a combination of arginine and calcium carbonate. The technology works through a natural process of dentine tubule occlusion attracting arginine and calcium carbonate to the dentine surface to plug open dentine tubules. With regular use, the Pro-Argin technology builds a long lasting protective barrier that acts like a seal against dentine hypersensitivity.

The new Colgate Sensitive Pro-Relief Sensitivity Relief Pen provides a flexible and easy solution for all patients who suffer from dentine hypersensitivity. It can be used on its own if you have patients who need a non-sensitivity toothpaste to address other dental concerns.

For more information on the Colgate Sensitive Pro-Relief range visit www.colgateprofessional.co.uk.

\section{Colouring opinions about digital scanning}

Align Technology's iTero Element intraoral scanner software has been upgraded to allow, among other things, scanning in colour.

The new iTero Element intraoral scanner, introduced in March 2015, is engineered to capture 6,000 frames per second, and the image sensor is designed to enable scan speeds which are up to 20 times faster than the former iTero scanner. The result is a real-time $3 \mathrm{D}$ visualisation of the digital scan which is ready in just minutes for viewing on a multi-touch screen.

The new software version 1.4 enhancements are designed to greatly enrich the user experience for specialists and GPDs performing orthodontic treatments and include:

SCAN IN COLOUR: It is now possible to scan in real-time in colour, which makes it easier for users to visually distinguish between gingival margins and tooth structure. The colour scan will also provide additional information to laboratory technicians when they work to emulate the shading of the original dentition when tackling restorative cases.

SCAN TIMER: A scanner timer tool is now upgraded so that it is possible to measure scan times in two different ways; the 'Elapsed Scan Time' is the cumulative time of the upper, lower and bite scans; whilst the 'Elapse Total Time' is the cumulative of the scan and processing time.

OPTIMISED SCANNING: With the new software upgrade, when scanning any orthodontic scan type there will be a visual representation of missing scan data. This will give users real-time scan feedback before processing the case, saving time at a later stage and optimising scan quality.

RESTORATIVE PRE-TREATMENT

SCANNING: With this new feature, dentists can scan the dentition before and after restorative preparation, allowing digital design of the treatment to match the original tooth shape and form, if desired, for even better patient comfort. This feature is enabled in the dental laboratory for customers using Exocad and 3Shape laboratory CAD software. The model and viewfinder have also been made larger for increased visibility and accurate scanning of the dentition, gingival margin and soft tissue.

For owners of the iTero Element intraoral scanner, there is no additional cost for the software upgrade. Visit www. itero.com or contact 08009171643.

\section{Fast and trackable handpiece repair}

Trigiene understands that no practice can afford to be without their dental handpieces. These are the backbone of every dental practice and one of the hardest working tools, withstanding the pressure of day-to-day workloads and repeated cleaning and sterilisation. This is why Trigiene's team of skilled and accredited technicians are on hand to quickly bring your valued handpieces back to their former glory.

With a fast 24-hour online trackable repair service, free insured collection and delivery, Trigiene Repair offers convenience, security and peace of mind. The 'no-obligation' online quotation system ensures the repairs are always competitively priced.

Simply book your repairs online or by phone and Trigiene's unique online tracking service will keep you fully informed of current and previous repair statuses, showing all your existing warranties too.

If you are looking for a handpiece upgrade, replacement, maintenance training, dental supplies, equipment or consumables then speak to Trigiene's expert sales team to discuss your requirements in detail or visit Trigiene Dental's online shop for some amazing special offers.

Book your handpiece repair today and receive your free voucher offer book. Call Trigiene on 01642442910 or visit www.dentalhandpiecerepairs.co.uk. 